\title{
VISITAS VIRTUAIS NAS UNIDADES NEONATAIS DURANTE PANDEMIA COVID-19: EXPERIÊNCIA DA EQUIPE DE SAÚDE
}

\section{VIRTUAL VISITS IN NEONATAL UNITS DURING THE COVID-19 PANDEMIC: EXPERIENCE OF THE HEALTH TEAM}

\author{
Rebeca Silveira Rocha ${ }^{1} *$ Anne Fayma Lopes Chaves ${ }^{2} *$ Mara Carolina Ribeiro Gomes $^{3} *$ \\ Maria do Socorro Leonacio ${ }^{4} *$ Myrna Araújo Cavalcante $^{5} *$ Priscila Magalhães de Medeiros $^{6}$ \\ * Naara Ingrid da Silva Sales ${ }^{7}$
}

\section{RESUMO}

Objetivo: relatar a experiência da equipe multiprofissional na realização de visitas virtuais entre recémnascidos internados em unidades neonatais e seus familiares durante a pandemia da Covid-19. Método: relato de experiência de profissionais de saúde que trabalham em unidades neonatais de uma maternidade em Fortaleza, Ceará, Brasil, sobre a realização de visitas virtuais entre recém-nascidos internados em unidades neonatais e seus familiares. A visita virtual ocorreu no período de abril a junho de 2020 através de videochamada por meio do aplicativo WhatsApp, sendo utilizado um tablet institucional. Resultados: durante as videochamadas, foi possível perceber a satisfação das mães ao olhar e dialogar com seus filhos, os quais se manifestavam por meio da movimentação, das expressões e reações diante da interação com sua genitora. A equipe multidisciplinar vivenciou momentos de emoção e satisfação ao poder manter vivo o vínculo mãe-filho. Considerações Finais: as visitas virtuais realizadas com auxílio da tecnologia possibilitaram à equipe multidisciplinar o incremento da aproximação mãe-filho, sendo uma experiência capaz de subsidiar outras instituições para o desenvolvimento de ações efetivas e humanizadas.

Palavras-chave: Unidade de Terapia Intensiva Neonatal; Recém-Nascido Prematuro; Infecções por Coronavirus; Relações Mãe-Filho; Equipe de Assistência ao Paciente.

\begin{abstract}
Objective: to report the experience of the multiprofessional team in carrying out virtual visits among newborns hospitalized in neonatal units and their families during the Covid-19 pandemic. Method: report of the experience of health professionals who work in neonatal units of a maternity hospital in Fortaleza, Ceará, Brazil, about the performance of virtual visits among newborns admitted to neonatal units and their families. The virtual visit took place from April to June 2020 via video call through the WhatsApp application, using an institutional tablet. Results: during the video calls, it was possible to perceive the mothers' satisfaction when looking and talking to their children, who manifested themselves through movement, expressions and reactions in the face of interaction with their mother. The multidisciplinary team experienced moments of emotion and satisfaction in being able to keep the mother-child bond alive. Final Considerations: virtual visits carried out with the aid of technology enabled the multidisciplinary team to increase the mother-child approach, being an experience capable of subsidizing other institutions for the development of effective and humanized actions.

Keywords: Neonatal Intensive Care Unit; Premature Newborn; Coronavirus infections; Mother-Child Relations; Patient Assistance Team.

\footnotetext{
${ }^{1}$ Universidade Federal do Ceará. Programa de Pós-Graduação em Enfermagem da UFC. Fortaleza, Ceará, Brasil. https://orcid.org/0000-0001-9825$\underline{6298}$

${ }^{2}$ Universidade da Integração Internacional da lusofonia afro-brasileira. Programa de Pós-Graduação em Enfermagem da Unilab. Redenção, Ceará, Brasil. https://orcid.org/0000-0002-7331-1673

${ }^{3}$ Maternidade Escola Assis Chateubriand - MEAC, Unidade de Terapia Intensiva Neonatal. Fortaleza, Ceará, Brasil. https://orcid.org/0000-00023041-7005

${ }^{4}$ Maternidade Escola Assis Chateubriand - MEAC, Unidade de Terapia Intensiva Neonatal. Fortaleza, Ceará, Brasil https://orcid.org/0000-0003$0031-0642$

${ }_{5}^{5}$ Maternidade Escola Assis Chateubriand - MEAC, Unidade de Terapia Intensiva Neonatal. Fortaleza, Ceará, Brasil. https://orcid.org/0000-00016162-9206

${ }^{6}$ Maternidade Escola Assis Chateubriand - MEAC, Unidade de Terapia Intensiva Neonatal. Fortaleza, Ceará, Brasil. https://orcid.org/0000-00031280-5183

${ }^{7}$ Universidade da Integração Internacional da lusofonia afro-brasileira. Programa de Pós-Graduação em Enfermagem da Unilab. Redenção, Ceará, Brasil. https://orcid.org/0000-0003-2619-5558
} 


\section{INTRODUÇÃO}

O mundo está vivenciando uma Emergência de Saúde Pública causada pelo vírus Severe Acute Respiratory Syndrome Coronavírus 2 (SARS-CoV-2), que ocasiona a doença respiratória Coronavírus 2019 (COVID-19). A pandemia já acometeu milhões de pessoas e é responsável por mais de 700 mil mortes em todo o mundo. No Brasil, existem mais de um milhão de casos confirmados até o momento, apresentando uma taxa de letalidade em torno de $4,0 \%{ }^{(1)}$.

Embora ainda não haja evidência consolidada da transmissão vertical do SARSCoV-2, recentemente pesquisas apontam para esta possibilidade ${ }^{(2)}$. Um estudo de caso publicado no Peru encontrou Reverse Transcription - Polymerase Chain Reaction (RT-PCR) positivo em neonato no primeiro dia de vida, mesmo tendo sido isolado logo ao nascimento da mãe que tinha swab positivo para SARS-CoV-2, sugerindo possível transmissão vertical ${ }^{(3)}$. Outra pesquisa realizou estudo histológico e de imunohistoquíminca que comprovam a transmissão placentária da viremia neonatal, resultando em manifestações neurológicas no recémnascido consistentes com a dos adultos ${ }^{(4)}$.

Além da possibilidade de transmissão vertical, outra preocupação é a contaminação de recém-nascidos por pessoas infectadas com SARS-CoV-2, uma vez que estudo aponta que, especificamente com relação à COVID19, as crianças menores de um ano têm taxas mais altas de complicações graves do que as crianças mais velhas $^{(5)}$.

Nesse contexto e devido a rápida disseminação do vírus, foram decretadas uma série de medidas para enfrentamento da COVID-19, dentre elas o distanciamento social, no qual é recomendado que a sociedade permaneça em suas residências, restringindo-se ao máximo o contato entre pessoas. Logo, o Ministério da Saúde determinou que as unidades neonatais não deviam ser fechadas nem reduzidas, no entanto, deveriam adotar cuidados com a prevenção de aglomerações, garantia do acesso às pessoas assintomáticas e que não tenham contato domiciliar com portadores de COVID-19, visando reduzir o fluxo de pessoas e minimizar os riscos de contaminação de pacientes e trabalhadores da saúde ${ }^{(6)}$.

Evidências apontam que a hospitalização de um filho em unidade neonatal é uma experiência estressante para os pais, o que gera sentimentos de medo e solidão diante do distanciamento e despreparo $^{(7)}$. Do contrário, o sentimento de prazer e competência quando recebem apoio da equipe de saúde deixa-as mais confiantes e empoderadas $^{(8)}$.

Nesse contexto, as visitas virtuais têm sido utilizadas por diversas instituições de saúde durante a pandemia do COVID-19, a qual tem como finalidade manter o vínculo e apoio psicológico ao paciente durante sua 
internação com a utilização de tecnologias disponíveis, visto que as visitas presenciais estão suspensas ${ }^{(9)}$. Sabendo que a comunicação envolve a relação entre uma pessoa e outra, tanto fisicamente como virtualmente, é reconhecido pelo Conselho Federal de Medicina a utilização de recursos virtuais, como WhatsApp, para ser usado na comunicação entre médicos e pacientes ${ }^{(10)}$.

A relevância da pesquisa reside no fato de que, em época de pandemia, é necessário pensar além de medidas de combate e prevenção. É preciso o desenvolvimento de estratégias que também trabalhem a saúde mental devido à carga emocional muito intensa, em que o acolhimento e a comunicação se constituem ações primordiais de humanização em saúde, fortalecendo o vínculo paciente e família. A socialização dessas estratégias irá subsidiar outras instituições para o desenvolvimento de ações efetivas e viáveis no que se refere à promoção da saúde materno-infantil durante esse momento específico, proporcionando uma atenção qualificada, que envolva o apoio emocional.

Desse modo, este estudo teve como objetivo relatar a experiência da equipe multiprofissional na realização de visitas virtuais entre recém-nascidos internados em unidades neonatais e seus familiares durante a pandemia COVID-19.

\section{DESCRIÇÃO DA EXPERIÊNCIA}

Tratou-se de um relato de experiência de profissionais de saúde na realização de visitas virtuais entre mães, e demais familiares, e recém-nascidos internados na Unidade Neonatal da Maternidade Escola Assis Chateaubriand, referência na atenção à saúde do recém-nascido no estado do Ceará no período de abril a junho de 2020. A referida instituição é um dos seis Centros de Apoio às Boas Práticas na atenção obstétrica e neonatal da Rede Cegonha no Brasil e sua Unidade Neonatal é composta por duas Unidades de Terapia Intensiva Neonatal (21 leitos) e duas Unidades de Cuidados Intermediários Convencionais (30 leitos). Além destas, uma unidade de isolamento foi temporariamente criada para internação de recém-nascidos suspeitos ou confirmados de COVID-19 comportando nove leitos no máximo.

O projeto de realização das visitas virtuais foi idealizado pela equipe de Assistentes Sociais e Psicóloga responsáveis pela Unidade Neonatal, que realizavam as chamadas e acompanhavam todo o processo dando o suporte emocional necessário à família, e contou com o apoio de toda a equipe multiprofissional, principalmente dos médicos, que avaliavam se o recém-nascido tinha estabilidade do quadro clínico para a realização da visita virtual, e da enfermagem, tanto Enfermeiras como Técnicos de Enfermagem, que possibilitava a realização da 
visita dando todo o suporte necessário, principalmente no contato direto ao recémnascido.

Antes da implementação do projeto, foi realizado um teste piloto no final de março de 2020. Após os devidos ajustes e organização da equipe, o início do projeto ocorreu logo no início de abril de 2020. No entanto, o quantitativo de videochamadas foi reduzindo gradativamente a partir da segunda metade de junho do mesmo ano, momento no qual iniciaram as visitas presenciais agendadas das mães às unidades neonatais, tornando a visita virtual restrita às mães impossibilitadas de se fazer presente.

A seleção dos recém-nascidos e sua mãe/familiar que participariam do projeto de visita virtual ocorreu de acordo com a solicitação da mãe ou familiar responsável ao Serviço Social ou demanda da equipe de médicos e enfermeiros da Unidade Neonatal a partir da necessidade da interação do recémnascido com a mãe, principalmente em casos de internação prolongada. As mães que manifestavam maior insegurança e dúvida no contato telefônico com a equipe médica também eram selecionadas para a videochamada, a partir da sinalização da equipe médica ao Serviço Social.

A visita virtual ocorreu através de uma videochamada por meio do aplicativo WhatsApp. Previamente, as mães eram contactadas pelas assistentes sociais e, quando manifestavam desejo de participação das visitas virtuais, era agendado com a mãe ou familiar responsável o melhor horário para a realização da mesma, articulando sempre com a equipe de saúde da Unidade Neonatal. No horário agendado, eram realizadas três tentativas no máximo de contato por videochamada com a mãe/familiar e, caso não obtivesse êxito, era feito um novo agendamento. No total, foram 35 tentativas de contato sem êxito.

A videochamada era realizada por meio de um tablet institucional, que possibilitava através de uma tela mais ampla, que o recém-nascido tivesse melhor visualização da mãe e/ou familiar, facilitando sua interação. Era possibilitado também que os sons das falas das mães e familiares fossem ouvidos pelo recém-nascido, sempre estimulando às mães e/ou familiares que conversassem com ele. Do mesmo modo, as mães também podiam ver seu filho, observar sua movimentação, suas expressões e reações, além de ouvir os sons que ele produzia.

Neste momento de videochamada, não eram permitidos questionamentos das mães aos profissionais de saúde sobre condições clínicas do recém-nascido, sempre ressaltando que aquele momento era único para contato dela com seu filho, para que possam interagir e estimular o vínculo entre ambos. Para informações sobre quadro clínico do recémnascido, destinou-se um horário da rotina na Unidade Neonatal para que médicos e/ou enfermeiros recebessem as ligações da mãe ou 
pai do recém-nascido internado para esclarecer esse tipo de informações.

As videochamadas tiveram uma duração de três a cinco minutos e eram realizadas em torno de sete a oito visitas virtuais por dia. Portanto, no mês de abril foram realizadas 106 videochamadas, em maio/20 123 e em junho/20 44.

\section{ANÁLISE DA EXPERIÊNCIA}

Frente à situação imposta pela pandemia do coronavírus, que restringiu o acesso das mães e pais dos recém-nascidos internados à Unidade Neonatal, os profissionais de saúde tiveram que inventar ou reinventar métodos, principalmente utilizando-se de recursos tecnológicos, garantido a segurança de todos os sujeitos envolvidos. A principal motivação era a consciência do prejuízo no vínculo afetivo entre mãe-filho, bem como vários relatos de angústias maternas de se manter à distância do seu filho e, ao mesmo tempo, medo de ser responsável pela contaminação dele pelo vírus.

Pesquisando iniciativas que pudessem minimizar o sofrimento das famílias diante desse distanciamento social, conhecemos a experiência de outros países com a videochamada dos pacientes adultos que estavam internados por Covid-19 e seus familiares. No Brasil, um grupo de profissionais de São Paulo com experiência em Cuidados Paliativos desenvolveu um documento com recomendações de como seria esta experiência, o qual utilizamos como guia para construção deste projeto de visitas virtuais, adaptando-o a nossa realidade com os recém-nascidos internados e suas famílias, tentando minimizar as alterações na dinâmica de serviço assistencial da Unidade Neonatal.

Sem a pretensão de substituir a presença constante da mãe e/ou pai nos cuidados com os bebês na unidade, as videochamadas funcionaram como uma forma de manter esse sentimento de proximidade e acompanhamento de seus filhos, inclusive facilitando também a interação da família com os cuidadores institucionais do recémnascido.

Foi visível a satisfação das mães em poder interagir, mesmo que virtualmente, com o seu bebê. Geralmente, este momento é compartilhado por outros membros da família e comunidade, sendo visto como a apresentação do bebê naquele contexto que, devido à hospitalização, o impede de ser vivido. Pai, mãe, irmãos, parentes e amigos compartilham com alegria e intensa emoção: aquele bebê exibido na tela e diz "eu existo". Pequenos movimentos são interpretados pelos expectadores, dando um colorido subjetivo à cena. O bebê, por sua vez, na maioria das vezes, reage aos sons familiares, mostrando que, de alguma forma, a experiência é registrada e sentida pela criança, mesmo naquelas bem prematuras. 
Os pais e familiares conversam com o bebê, contando como estão, emocionam-se, falam da vontade de estarem perto e da espera de todos pela chegada dele em casa. Ao mesmo tempo, percebemos que este movimenta-se, abre os olhos e tenta interagir ao ouvir a voz dos pais. É surpreendente e emocionante para toda equipe. As videochamadas nunca são iguais, ainda que com o mesmo bebê, pois as reações deles e dos pais/familiares são sempre inesperadas.

Durante a videochamada, percebemos que somos as atravessadoras, damos voz a estes bebês, respondemos através do "manhês" o que aquelas mães e famílias vão dando significados a este momento tão difícil de vivenciar.

A emoção corre frouxa, as mãos tremem segurando o tablet pela sensação de ver as famílias apresentando aos seus bebês seus novos lares, pelos rostos expressando suas emoções, pela resposta dos bebês que param de chorar quando escutam as vozes, que fazem gestos que dão encantamento para quem está do outro lado. Pequenos gestos deles dão uma conotação visível de realização afetiva desse encontro com esta família, que sempre demonstra gratidão pelo momento vivenciado.

Realizar as videochamadas requer dedicação, disponibilização de tempo, articulação com a dinâmica da Unidade e envolvimento de vários profissionais que prestam assistência direta ou indireta ao recém-nascido. No entanto, para todos nós, o que prevalece é o nosso dever de fazer o que estiver ao alcance das nossas mãos para que estas distâncias se encurtem, que o vínculo mãe-filho se mantenha vivo, que as fantasias deem lugar à imagem real e que, mesmo que a imagem os mostre incubadoras, aparelhos e barulhos dos equipamentos, o que percebemos que estas famílias veem são seus bebês, somente os seus bebês.

Vale ressaltar que, durante a realização das visitas virtuais, tivemos dificuldade com o tablet, que possui baixa resolução da imagem, como também as limitações de muitas famílias ao acesso à internet de qualidade, ou até mesmo ausência de acesso, que dificulta e até impossibilita a realização da videochamada. Em alguns casos, enviamos a foto do bebê, para amenizar essa dificuldade.

O recurso não pretende e nem poderia substituir a presença das mães e pais na Unidade Neonatal. A estratégia utilizada tem como principal limitação a impossibilidade de favorecer ao toque, a baixa resolução da imagem disponibilizada pelo equipamento e a necessidade de acesso à internet de qualidade, indisponível à realidade de muitas das famílias. Além disso, a duração da chamada é reduzida, comparado à permanência constante e por tempo indeterminado dos pais antes da pandemia, porém alivia a angústia dos pais por conseguirem demonstrar tanto carinho e amor ao seu filho em uma ligação e por ver 
que seus filhos estão sendo bem cuidados por toda a equipe.

Diante da rápida disseminação do vírus e da necessidade de adequações institucionais para prevenção da transmissão, é evidente o impacto da pandemia nos serviços de assistência ao recém-nascido. A restrição de visitas impede a ligação entre mãe e bebê e limita a promoção dos cuidados centrados na família pela Unidade Neonatal, o que fragiliza práticas facilitadoras de vínculo conquistadas ao longo do tempo ${ }^{(11)}$.

Nesse contexto, é fundamental a atuação da equipe multidisciplinar no acolhimento aos pais, fornecendo orientações adequadas sobre os cuidados e contribuindo com o enfrentamento de medos, angústias e dúvidas. Assim, os profissionais estão buscando estratégias de intervenção na rotina de cuidados em unidades neonatais voltadas para a família ${ }^{(12)}$.

Anterior a pandemia COVID-19, as evidências já apontavam a necessidade da utilização de diversos meios para favorecer a comunicação dos familiares de RN internados em UTIN, sendo dado ênfase aos recursos tecnológicos para disponibilizar informações, satisfazendo as necessidades informativas da família, resultando em cuidado humanizado diante do momento de fragilidade vivenciado $^{(13)}$.

No panorama da pandemia da COVID-19, a telemedicina vem sendo globalmente utilizada por meio da telecomunicação, as quais visam repassar informações de saúde para diagnóstico, terapia de tratamento e educação em saúde, demonstrando ser uma alternativa viável, aceitável e eficaz ${ }^{(14)}$.

No Brasil, um grupo de profissionais de São Paulo com experiência em Cuidados Paliativos desenvolveram um guia de recomendações práticas para comunicação e acolhimento em diferentes cenários da pandemia. A proposta da visita virtual visa manter o vínculo e o apoio psicológico ao paciente e à família durante sua internação, sendo importante a presença de um profissional de psicologia ou serviço social para acompanhamento ${ }^{(9)}$.

$\mathrm{O}$ uso do aparelho celular nas unidades neonatais sempre foi restrito, porém hoje é vista como uma importante ferramenta nesse momento delicado, visando encurtar a distância entre a família e o bebê. Uso de mensagens gravadas ou lidas pela equipe de saúde podem ser enviadas, favorecendo o envolvimento de outros familiares (avós e irmãos), registros de vídeos e fotos, descrições sobre o comportamento do bebê, seu jeito e sua rotina podem ser enviados para os pais também como forma de amenizar a dor e promover o vínculo ${ }^{(15)}$.

No momento dessas interações, foi possível utilizar o "manhês", que são padrões prosódicos característicos da fala da mãe, um tipo de diálogo que a mãe ou o cuidador adulto utiliza quando se dirige ao bebê, como 
sussuros, duração prolongada de certas palavras que as tornam mais lentas e sonoras e aumento da frequência, que a faz mais aguda, o que favorece o estímulo e afeto durante a comunicação ${ }^{(16)}$.

Evidência científica também aponta o efeito positivo da exposição a voz materna na resposta fisiológica dos bebês prematuros internados em Unidades Neonatais. Após exposição de uma gravação de áudio da voz materna durante 15 minutos por três dias consecutivos, 20 bebês prematuros apresentaram aumento na saturação de oxigênio e melhora nos parâmetros de frequência cardíaca e respiratória, sendo estimulado o desenvolvimento dessas estratégias nas unidades neonatais ${ }^{(17)}$.

Apesar das mães vivenciarem os sentimentos de tristeza, impotência e medo da morte do seu filho durante a hospitalização nas Unidades Neonatais, ao ver a recuperação clínica do prematuro, é amenizado o sofrimento materno, a mulher sente-se mais confiante e fortalecida para enfrentar a situação. Além disso, a puérpera desenvolve confiança na equipe de saúde e na própria estrutura da Unidade Neonatal ${ }^{(18)}$.

As intervenções digitais de saúde estão desempenhando papel significativo na assistência à saúde diante da pandemia da COVID-19. No entanto, é evidente as lacunas apresentadas por essas tecnologias quanto a fragilidade no relacionamento interpessoal, no repasse de informações as quais podem gerar preocupações desnecessárias e nos custos adicionais $^{(19)}$.

A natureza do trabalho da equipe de neonatologia no cuidado ao RN prematuro é originalmente caracterizada como emocionalmente estressante diante da sua assistência a bebês graves e seus pais fragilizados no aspecto emocional. Contudo, esses profissionais percebem seu papel como essencial no apoio e desenvolvimento do vínculo mãe-bebê, ainda que seja uma assistência emocionalmente desafiadora ${ }^{(20)}$.

\section{CONSIDERAÇÕES FINAIS}

As visitas virtuais realizadas com auxílio da tecnologia possibilitaram a equipe multidisciplinar o incremento da aproximação dos RN internados nas Unidades Neonatais com seus pais no contexto da pandemia COVID-19, sendo uma oportunidade de promover o vínculo entre mãe e filho, favorecendo a saúde materno-infantil.

No decorrer das videochamadas, foi possível perceber a satisfação das mães ao olhar e dialogar com seus filhos, os quais se manifestavam por meio da movimentação, das expressões e reações diante da interação com sua genitora. Além disso, essa estratégia tecnológica gerou na equipe multidisciplinar da unidade neonatal um sentimento de gratidão, ao vivenciar a emoção de poder manter vivo vínculo mãe-filho em um momento tão delicado. 
Este relato de experiência traz informações e direcionamentos para uma prática humanizada nas unidades neonatais diante do contexto específico. A divulgação e o consumo crítico dessa experiência são imprescindíveis para subsidiar outras instituições para o desenvolvimento de ações efetivas e humanizadas com vistas à melhoria da qualidade do cuidado prestado.

\section{REFERÊNCIAS}

1. Worldometer. Real time world statistics. [acessado 2020 July 07]. Available from:

https://www.worldometers.info/coronavirus/

2. Zhu N, Zhang D, Wang W, Li X, Yang B, Song J, et al. A novel Coronavirus from patients with pneumonia in China, 2019. N Engl J Med [Internet].2020 [cited May 28, 2020];382:727-33.

https://doi.org/10.1056/NEJMoa2001017

3. Alzamora MC, Paredes T, Caceres D, Webb CM, Valdez LM, Rosa ML. Severe COVID-19 during Pregnancy and Possible Vertical Transmission. Am J Perinatol [Internet]. 2020 [cited July 07, 2020];37:8615. https://doi.org/10.1055/s-0040-1710050

4. Vivanti AJ, Vauloup-Fellous C, Prevot S, Zupan V, Suffee C, Do Cao J, et al. Transplacental transmition of SARS-CoV-2 infection. Nature Communications. Nature Communications [Internet].2020 [cited Ouc 26, 2020];23(3572):1-10. https://doi.org/10.1038/s41467-020-17436-6

5. Dong Y, Mo X, Hu Y, Qi X, Jiang F, Jiang Z, et al. Epidemiological characteristics of 2143 pediatric patients with 2019 coronavirus disease in China. Pediatrics [Internet].2020 [cited July 07, 2020];145(6):e20200702.

https://doi.org/10.1542/peds.2020-0702

6. Ministério da Saúde (BR). Secretaria de Vigilância em Saúde (SVS): Guia de Vigilância Epidemiológica do COVID-19. Painel Coronavírus. Brasília: Ministério da Saúde; 2020.

7. Kegler JJ, Neves ET, Silva AM, Jantsch LB, Bertoldo CS, Silva JH. Stress in Parents of Newborns in a Neonatal Intensive Care Unita. Esc Anna Nery [Internet].2019 [cited Ouc 28, 2020];23(1):e20180178:1-6. https://doi.org/10.1590/2177-9465-EAN2018-0178

8. Mahnaz J, Hadi H, Reza N, Mohammad A, Lisa MC. Mothers' Emotional Experiences Providing Care for Their Infants Within the Culture of an Iranian Neonatal Unit. Adv. Neonatal Care [Interne].2018 [cited July 07, 2020];18(4):E3-E12. https://doi.org/10.1097/ANC.0000000000000 530

9. Crispim D, Silva MJP, Cedotti W, Câmara M, Gomes SA. Visitas Virtuais durante a pandemia do COVID-19. Recomendações práticas para comunicação e acolhimento em diferentes cenários da pandemia. 2020. Belo Horizonte, Minas Gerais. Available from: https://ammg.org.br/wpcontent/uploads/Visitas-virtuais-COVID19.pdf

10. Nazareth RT, Almeida JJG, Bastos AT. Utilização do Whatsapp e o Parecer CFM $\mathrm{N}^{\mathrm{o}}$ 14/2017. Revista UNIB [Internet].2020 [cited July 07, 2020];19:17-22. Available from: http://seer.unib.br/index.php/rev/index

11. Abdul-Mumin A, Faith A; Abdulai A; Albrecht J. Maintaining quality newborn care in Ghana amid the COVID-19 pandemic. Pan Afr Med J [Internet].2020 [cited July 02, 2020];35(Suppl

2):6. https://doi.org/10.11604/pamj.2020.35.6.2279 7 
12. Mesquita DS, Naka KS, Kawamura APS, Schmidt AS. Nursing care at the neonatal intensive care unit (ICU) according to parent-child binomial: integrative literature review study. REAS/EJCH [Internet].2020 [cited July 02, 2020];11(13):e980. https://doi.org/10.25248/reas.e980.2019

13. Lima VF, Mazza VA. Information needs of families on the health/disease of preterm infants in a neonatal intensive care unit. Texto \& Contexto Enfermagem [Internet].2019 [cited Oct 28, 2020];28(e20170474). https://dx.doi.org/10.1590/1980-265X-TCE2017-0474

14. Zhen H, Nian L, Dajiang L, Junhua L, Bing L, Weixi X, et al. Telemedicine During the COVID-19 Pandemic: Experiences From Western China. J Med Internet Res [Internet].2020 [cited July 01, 2020];22(5):e19577. https://doi.org/10.2196/19577

15. Morsch DS, Custódio ZAO, Lamy ZC. Psycho-emotional care in a neonatal unit during the Covid-19 pandemic. Rev Paul Pediatr [Internet].2020 [cited Juy 01, 2020];38:e2020119.

https://doi.org/10.1590/1984-

0462/2020/38/2020119

16. Splendore KM, Constantini AC, Da Silva KCB. Investigation of prosody and language in mother-baby interaction. Working Papers em Linguística [Internet].2019 [cited Oct 28, 2020];20(1):172-188. https://doi.org/10.5007/1984-

8420.2019v20n1p172

17. Sajjadian N, Mohammadzadeh M, Taheri PA, Shariat M. Positive Effects of Low Intensity Recorded Maternal Voice on
Physiologic Reactions in Premature Infants. Infant Behav Dev [Internet].2017 [cited July 02 , 2020];46:59-66. https://doi.org/10.1016/j.infbeh.2016.11.009

18. Da Silva KC, Kerber NPC, Silva CSG, Christoffe MM, Carvalho ESS, Passos SSS, et al. Maternal experiences during hospitalization of premature newborn. Ver Soc Bras Enferm Ped [Internet].2019 [cited July $\quad 01, \quad 2020] ; 19(1): 7-15$. http://dx.doi.org/10.31508/16763793201900002

19. Sohini S, Sarbadhikari SN. The global experience of digital health interventions in COVID-19 management. Indian J Public Health [Internet].2020 [cited July 02, 2020]; 64(Supplement):S117-S124.

https://doi.org/10.4103/ijph.IJPH_457_20

20. Twohig A, Reulbach U, Fiquerdo R, McCarthy A, McNicholas F, Mollov EJ. Supporting preterm infant attachment and socioemotional development in the neonatal intensive care unit: staff perceptions. Infant Ment Health J [Internet].2016 [cited July 01, 2020];37(2):160-71.

https://doi.org/10.1002/imhj.21556

\section{Autor Correspondente}

Rebeca Silveira Rocha

Maternidade Escola Assis Chateubriand - MEAC, Unidade de Terapia Intensiva Neonatal. E-mail: bekinharocha@hotmail.com

Rua Coronel Nunes de Melo S/N Rodolfo Teófilo CEP: 60.430-270 Fortaleza - Ceará Brasil Telefone: (85) 33668501

Submissão: 2021-07-15

Aprovado: 2021-10-14 